\title{
The impact of the UK Quality and Outcomes Framework (QOF) on general practice quality of care
}

\section{Background}

In 2004 the United Kingdom government introduced a pay for performance scheme for general practices with indicators covering chronic disease management, practice organisation and patient experience. In 2006, 7 new clinical areas such as dementia and chronic kidney disease were included and 2 new indicators of patient access to care. Payments make up approximately $25 \%$ of general practitioners' income and 99,6\% participate, although the scheme is voluntary. In April 2009 the National Institute for Health and Clinical Excellence became responsible for overseeing the development of QOF indicators along with an external contractor led by staff at NPCRDC, University of Manchester.

\section{Methods}

Study 1) [2,3]: Interrupted time series analysis of quality of care in 42 representative general practices, with data collected before (1998 and 2003) and after (2005 and 2007) the scheme. At each time point, data for asthma, diabetes and coronary heart disease were extracted from medical records, and data on access, continuity of care and inter-personal aspects of care collected from patient questionnaires.

Study 2) [1]: Interviews with 21 family doctors and 20 nurses in 22 nationally representative practices across England between February-August 2007.

\section{Results}

Study $1[2,3]$ : Immediately following introduction of the scheme (2003-2005) the rate of quality improvement increased for asthma and diabetes $(\mathrm{p}<0.001)$ but not heart disease. In the longer-term (2005-2007), improvement rates slowed for all 3 conditions $(\mathrm{p}<0.001)$ and care for non-incentivized aspects of asthma and heart disease care declined. Compared to the pre-incentives period the improvement rate after 2005 was no different for asthma or diabetes, and reduced for heart disease $(p=0.02)$. No significant changes were seen in patients' reports of access to care or inter-personal aspects of care. Previously constant levels of continuity of care showed a step reduction following the introduction of pay for performance $(p<0.001)$ and then continued at the new lower level.

Study 2 [1]: Participants believed the financial incentives had been sufficient to change behaviour and to achieve targets and that it is not necessary to align targets to professional priorities and values to obtain behaviour change; although doing so enhances enthusiasm and understanding. Participants agreed that the aims of the pay for performance scheme had been met in terms of improvements in disease specific processes of patient care and physician income as well as improved data capture. However, it had also led to unintended effects such as the emergence of a dual QOF-patient agenda within consultations, potential deskilling of doctors due to an enhanced role for nurses in managing long-term conditions, a decline in personal/relational continuity of care between doctors and patients, resentment by team members not benefiting financially from payments and concerns about an ongoing culture of performance monitoring in the UK.

\section{Conclusions}

Against a background of increasing quality before the scheme was introduced, the pay for performance scheme accelerated improvements in quality for 2 of 3 chronic conditions in the short-term. However, once targets had been reached quality improvement slowed and for 2 conditions non-incentivized care declined. Continuity of care was reduced following the introduction of the scheme. The QOF scheme may have achieved its declared objectives of improving disease specific processes of patient care through the achievement of clinical and organizational targets and increased physician income. However, there is some evidence that it has changed the dynamic between doctors and nurses and the nature of the practitioner-patient consultation.

The presentation at the Potsdam conference will describe the QOF and evidence about its impact on quality of care. It will conclude by describing the new system for developing and piloting indicators, led by NICE, in the UK.

Author's declaration: The author is working with The National Institute of Health and Clinical Excellence (NICE) to support the ongoing development of indicators for the Quality and Outcomes Framework in the United Kingdom.

References

1 Campbell SM, McDonald R, Lester $H$. The experience of pay for performance in English family practice: A qualitative study. Annals of Family Medicine 2008; 6: 228-234

2 Campbell SM, Reeves D, Kontopantelis E, Sibbald B, Roland $M$. Impact of pay-for-performance on quality of English primary care: interrupted time series analysis. New England Journal of Medicine 2009; 361: 368-378

3 Campbell S, Reeves D, Kontopantelis E et al. Quality of primary care in England with the introduction of pay for performance. New England Journal of Medicine 2007; 357: 181-190

\section{S. Campbell}

Qualitätsmanagement

Keywords

pay for performance

Drimary care

quality of care

\section{Schlüsselwörter}

Leistungsorientierte Vergütung

- ambulante Versorgung

Versorgungsqualität

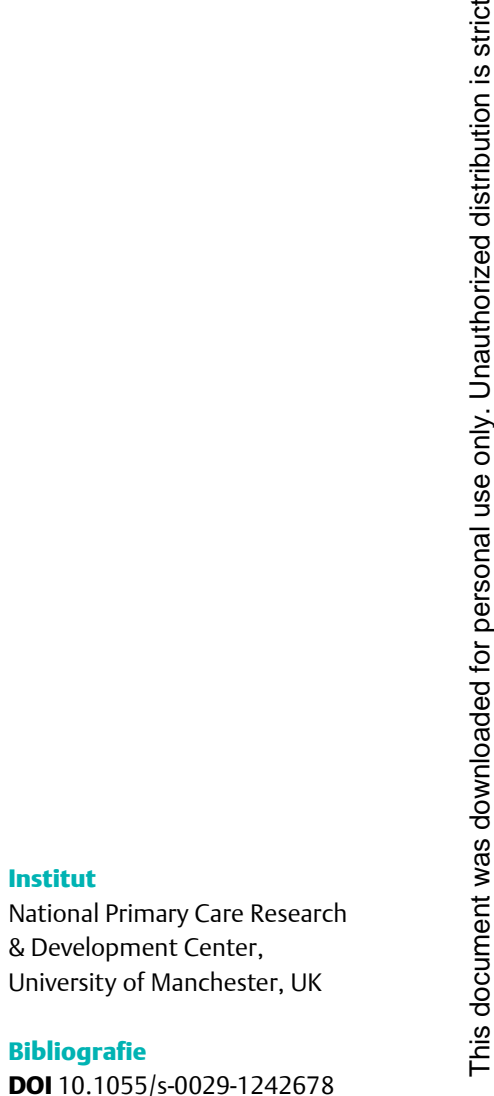

Dtsch Med Wochenschr 2009;

134: S323 - (c) Georg Thieme

Verlag KG Stuttgart - New York . ISSN 0012-0472

\section{Korrespondenz}

\section{Dr. Stephen Campbell}

Senior Research Fellow NPCRDC, University of

Manchester

Williamson Building

Oxford Road

Manchester

M13 9PL

Tel. +44 (0) 161275 7655-7601

eMail stephen.campbell@

manchester.ac.uk 